Volume 10, No.4, July - August 2021

International Journal of Advanced Trends in Computer Science and Engineering

Available Online at http://www.warse.org/IJATCSE/static/pdf/file/ijatcse131042021.pdf

https://doi.org/10.30534/ijatcse/2021/131042021

\title{
Pneumonia Disease Detection Using Deep Learning Methods from Chest X-Ray Images: Review
}

\author{
Shivanshu Katoch ${ }^{1}$, Saurabh Sharma ${ }^{2}$, Dr R.P.P Singh ${ }^{3}$ \\ ${ }^{1}$ Sri Sai University Palampur, India, katochshiva1997@gmail.com \\ ${ }^{2}$ Sri Sai University Palampur, India, saurabh23frmsnr@gmail.com \\ ${ }^{3}$ Sri Sai University Palampur, India, raminder_212003@ rediffmail.com
}

\begin{abstract}
Pneumonia is an infection-related condition under which the bronchi get damaged and clogged, decreasing oxygen diffusion and causing coughing and difficulty breathing. It can cause a range of symptoms, but it is more common in vulnerable populations. Pneumonia is the leading risk factor for mortality around the globe. Annually, pneumonia kills a significant percentage of youngsters around the globe. In 2016, an estimated one million cases of bronchitis were confirmed in kids under the age of five, with 880,000 deaths. The causes and symptoms of pneumonia are explained in this paper. There are several challenges occurs during detection of pneumonia from $\mathrm{x}$-ray images. Some challenges are explained in this paper. The comparison of existing method of pneumonia detection and classification is depicted with limitations. Various deep learning based architectures are discussed in this paper. The pre-trained models based on deep learning framework such as InceptionV-4, CNN, ResNet50, VGG16, VGG19.
\end{abstract}

Key words : Deep learning, Pneumonia, ResNet50, X-ray images.

\section{INTRODUCTION}

Pneumonia is a lung infection that accounts for 18 percent including all hospitalizations in children between the ages of 5. Furthermore, pneumonia affects around two million individuals over the world each year, with mortality a possibility even though no changes are made. Timely identification of pneumonia is critical. For minimize misinterpretation, a prompt diagnosis via a trained physician employing chest $\mathrm{X}$-rays is necessary [1].

Chest X-rays seem to be the most common and useful method of diagnosing pneumonia. Similarly, there is a scarcity of radiological professionals, particularly in low-resource roads and rural areas, resulting in lengthier delays for evaluations and a higher number of deaths [4]. Due to the extreme characteristics of chest X-ray computer vision, pneumonia treatments are frequently ambiguous and can also be mistaken for other illnesses with mild problems, like dullness, cavities, and pulmonary hypertension. As either a result, chest X-rays aren't as effective for detecting illnesses. Pneumonia effects range from moderate to serious, regardless of the type of bacteria that caused the illness, as well as aging and general wellbeing. Minor common symptoms are cold, flu but sometimes these symptoms become life threatening disease [14].

- Breathing Difficulties

- During breathing chest pain occur

- Phlegm during cough

- Fatigue

- Nausea

- Diarrhea and vomiting

- Breath shortness

- Sweating

- Shaking chills

Causes of Pneumonia [15] there are various mild and risky causes of pneumonia. Difficulties in breathing can cause pneumonia. The various causes of pneumonia are depicted in Table 1.

Table 1: Types and causes of pneumonia

\begin{tabular}{|l|l|c|}
\hline $\begin{array}{l}\text { Sr. } \\
\text { No. }\end{array}$ & Type of pneumonia & Causes \\
\hline 1. & Viral pneumonia & $\bullet$ Flu \\
& & $\begin{array}{c}\text { RSV (Respiratory } \\
\text { syncytial Virus) }\end{array}$ \\
& & - Common cold \\
\hline 2. & Fungal pneumonia & $\begin{array}{c}\text { - Weak immune } \\
\text { system }\end{array}$ \\
& & $\begin{array}{c}\text { Pneumocystis } \\
\text { jirovecii }\end{array}$ \\
& & $\bullet$ Histoplasmosis \\
\hline
\end{tabular}

A chest X-ray is a medical imaging procedure that involves introducing the chest to exposure for a slight specified period of time to obtain an image of the chest and its major organs. An X-ray material is formed against by the individual just on reverse side of the lens, which emits a quite low beam of medication [5]. The chest X-ray image is shown in figure1. 


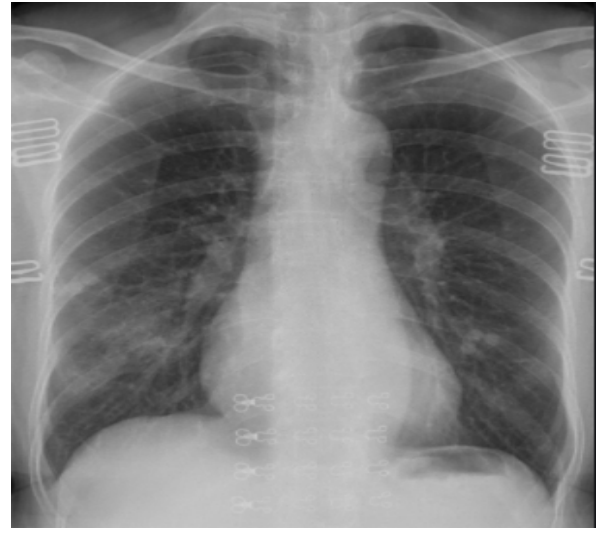

Figure 1: Chest X-ray image [5]

The main Challenges are; insufficient predictive power in pneumonia clinical characteristics often contributes to an underestimating of the impact of treatments affecting pneumonia. The number of monitoring sessions and indeed the deployment of an active or passive monitoring technique may result in unnecessary cases or tilt detection programs favoring weaker events. In some cases, mild symptoms can affect the most in pneumonia illness.

For advanced techniques, detection of mild symptoms is also hard to detect. Systems work on current information does not provide efficient results. Images scarcity reduced the accuracy results of the system.

In this paper, pneumonia and causes of pneumonia are mentioned in section 1 . The various pneumonia detection and classification techniques are reviewed in section 2. A brief comparison table is also depicted in literature survey. Deep Learning approaches of Pneumonia detection with chest $\mathrm{x}$-ray images are explained in section 3. A brief conclusion of paper is described in section 4 .

\section{RELATED WORK}

Rajasenbagam, et al. [16] proposed a deep CNN (Convolutional Neural Network) based method for the identification of Pneumonia disease in the chest through $\mathrm{X}$-ray frames of the lung. A dataset of pneumonia Chest X-rays encompassing 12,000 frames of affected and non-infected chest X-ray was used to train the proposed Deep CNN architectures. The Chest X-ray 8 dataset was used for pre-processing stage and create the information. To increase the number of frames inside each category, matrix factorization algorithms were employed. The modified photos were created using fundamental modification techniques and DCGAN termed as Deep Convolutional Generative Adversarial Network was used. The suggested Deep CNN model was built using the VGG19 connectivity. Throughout the unknown chest X-ray images, the proposed Deep CNN model had a predictive performance of $99.34 \%$. The suggested shallow CNN's results were compared with classification algorithms including VGG16Net, AlexNet, and InceptionNet. The comparative findings demonstrate that the suggested Shallow CNN model outperformed other strategies in terms of recognition accuracy. Hashmi et al. [6] proposed an effective approach for detecting pneumonia associated with digital chest X-ray frames, that would help clinicians make better decisions. The balanced estimations of deep learning models such as InceptionV3, ResNet18, MobileNetV, Xception, and DenseNet121 were combined in a revolutionary methodology based on the maximum classification. For the extension of the classification model, limited matrix factorization approaches were used. Most of the specific components were outperformed by the suggested balanced predictor. Ultimately, the analysis had been performed mostly based on classification accuracy and also based on such AUC value. Varshni et al. [2] developed an autonomous tool for classifying pneumonia to diagnose the condition as soon as possible, especially in rural places. CNN had received a lot of attention for illness categorization contributed to the quality of deep learning algorithms in evaluating healthcare imagery. Furthermore, image processing activities benefit greatly from features obtained using pre-trained neural networks on large amounts of data. The effectiveness of pre-trained $\mathrm{CNN}$ architectures was used as functionality reported by different classification models for both the identification of normal and anomalous chest X-Rays is evaluated in. The authors were used analysis to get the best Cnn architecture for the classification. The survey results presented that using fully convolutional Cnn architectures and automated classification algorithms to identify chest X-ray frames, particularly to diagnose Pneumonia, could be highly advantageous. Chouhan, V., et al. [7] designed a novel feature selection architecture for the identification of pneumonia and used the notion of learning algorithms. Throughout the architecture, frame attributes were retrieved and utilizing various pre-trained deep neural networks. The ImageNet pre-trained network was used for efficient outcomes. The authors had built five distinct simulations and compared their results. The authors had suggested a prediction method that combined outcomes across all methods for improving, outperforming control variables, and achieving efficient pneumonia detection accuracy. On different datasets from GWCMC termed as (Guangzhou Women and Children's Medical Center), the proposed system hybrid learning had achieved an accuracy of 96.4 percent and a recall of 99.62 percent. Acharya et al. [8] used a deep Siamese-based computational framework and proposed an automatic identification of pneumonia from images of the chest. The authors analyzed that, there were approaches that rely primarily on the transferable learner or classic customized procedures to categorize the pneumonia condition. To analyze or identify the issue, deep Siamese networks exploit the symmetric structure of the two input images. Every X-ray scan of the lungs was divided into two segments, which were then fed through into the system, and compared the symmetric structure as well as the quantity of illness dispersed over these two regions. To build and evaluate the proposed system for autonomous identification of multiple types of pneumonia infection, the authors were used the Kaggle dataset. The proposed methodology may aid medical practitioners in quickly diagnosing pneumonia using X-ray images. Elshennawy et al. [9] designed a reliable and 
efficient methodology for the detection and classification of pneumonia. The pre-trained (DL) deep learning approach utilized, four distinct versions were formulated for better results. The various DL-based networks such as CNN (Convolutional Neural Network), ResNet152V2, LSTM (Long Short-Term Memory), and MobileNetV2 were compared. The presented techniques were applied and assessed in the Python platform, and they were contrasted to other contemporary works in the domain. The suggested DL architecture enhanced the precision rate, accuracy, recall, AUC and, F1-score. The ResNet152V2 model surpasses other previously presented research, as evidenced by the findings.
Furthermore, the other experimental results, LSTM-CNN $\mathrm{CNN}$, had achieved outcomes in the recall, F1-score, accuracy, AUC, and, precision was more than 91 per cent which was higher than the other launched modeling techniques.

Table 2 depicted the various existing techniques for the detection of Pneumonia.

Table 2: Various Existing Techniques of Pneumonia Detection Based On Deep Learning Methods

\begin{tabular}{|c|c|c|c|c|c|c|}
\hline Author's & Year & $\begin{array}{l}\text { Proposed } \\
\text { method }\end{array}$ & $\begin{array}{l}\text { Compared } \\
\text { techniques }\end{array}$ & Dataset & $\begin{array}{l}\text { Performance } \\
\text { Metrics }\end{array}$ & Issues and Gaps \\
\hline $\begin{array}{l}\text { Rajasenbagam } \\
\text {, et al. [16] }\end{array}$ & 2021 & $\begin{array}{l}\text { Deep CNN } \\
\text { with } \\
\text { pre-trained } \\
\text { classifier } \\
\text { model }\end{array}$ & $\begin{array}{l}\text { VGG-16 Net, } \\
\text { AlexNet, } \\
\text { Inception V3 } \\
\text { Net }\end{array}$ & $\begin{array}{l}\text { Chest } \\
\text { x-ray8 } \\
\text { dataset }\end{array}$ & $\begin{array}{l}\text { Accuracy } 99.34 \% \\
\text { Recall } 98.6 \\
\text { Precision } 100\end{array}$ & $\begin{array}{l}\text { Low prevalence } \\
\text { at initial stages of } \\
\text { the illness. }\end{array}$ \\
\hline $\begin{array}{l}\text { Hashmi et al. } \\
\text { [6] }\end{array}$ & 2020 & $\begin{array}{l}\text { Deep } \\
\text { learning-base } \\
\mathrm{d} \text { approach for } \\
\text { pneumonia } \\
\text { detection }\end{array}$ & $\begin{array}{l}\text { Inception V3, } \\
\text { ResNet18, } \\
\text { MobileNetV, } \\
\text { Xception, and } \\
\text { DenseNet121 }\end{array}$ & $\begin{array}{l}\text { Pneumonia } \\
\text { dataset } \\
\text { Guangzhou } \\
\text { Women and } \\
\text { Children's } \\
\text { Medical } \\
\text { Center } \\
\end{array}$ & $\begin{array}{l}\text { Accuracy } 98.43 \% \\
\text { Precision } 98.26 \% \\
\text { Recall 99\% } \\
\text { AUC Score } 99.76 \%\end{array}$ & $\begin{array}{l}\text { Work only on } \\
\text { current } \\
\text { information of } \\
\text { patients }\end{array}$ \\
\hline $\begin{array}{l}\text { Varshni et al. } \\
\text { [2] }\end{array}$ & 2019 & $\begin{array}{l}\text { CNN based } \\
\text { framework }\end{array}$ & $\begin{array}{l}\text { XCeption, } \\
\text { VGG-19, } \\
\text { ResNet-50 }\end{array}$ & $\begin{array}{l}\text { Chest } \\
\text { radio-graph } \\
\text { s dataset }\end{array}$ & AUC Score $80.02 \%$ & $\begin{array}{l}\text { Time-consuming } \\
, \quad \text { High } \\
\text { computational } \\
\text { time }\end{array}$ \\
\hline $\begin{array}{l}\text { Chouhan, V., } \\
\text { et al. [7] }\end{array}$ & 2020 & $\begin{array}{l}\text { Deep } \\
\text { learning-base } \\
\text { d architecture } \\
\text { with } \\
\text { ImageNet } \\
\text { feature } \\
\text { extractor }\end{array}$ & $\begin{array}{l}\text { AlexNet } \\
\text { ResNet18 } \\
\text { DenseNet121 } \\
\text { GoogLeNet } \\
\text { InceptionV3 }\end{array}$ & $\begin{array}{l}\text { GWCMC } \\
\text { termed as } \\
\text { (Guangzhou } \\
\text { Women and } \\
\text { Children's } \\
\text { Medical } \\
\text { Center) } \\
\end{array}$ & $\begin{array}{l}\text { Accuracy 96.4\% } \\
\text { Recall 99.62\% }\end{array}$ & $\begin{array}{l}\text { The scarcity of } \\
\text { images reduced } \\
\text { the accuracy } \\
\text { results }\end{array}$ \\
\hline $\begin{array}{l}\text { Acharya et al. } \\
{[8]}\end{array}$ & 2020 & $\begin{array}{l}\text { Deep } \\
\text { learning-base } \\
\mathrm{d} \text { framework }\end{array}$ & - & $\begin{array}{l}\text { Kaggle } \\
\text { dataset }\end{array}$ & AUC Score $82.2 \%$ & $\begin{array}{l}\text { Inefficient results } \\
\text { on challenging } \\
\text { frames }\end{array}$ \\
\hline $\begin{array}{l}\text { Elshennawy et } \\
\text { al. [9] }\end{array}$ & 2020 & $\begin{array}{l}\text { Deep } \\
\text { network-based } \\
\text { Pneumonia } \\
\text { detection }\end{array}$ & $\begin{array}{l}\text { MobileNetV2, } \\
\text { ResNet152V2 }\end{array}$ & $\begin{array}{l}\text { Kaggle } \\
\text { dataset }\end{array}$ & $\begin{array}{ll}\text { Accuracy } & 99.22 \%, \\
\text { AUC } & 99.77 \%, \\
\text { precision } & 99.43 \%, \\
\text { recall } 99.44 \%\end{array}$ & $\begin{array}{l}\text { High } \\
\text { computational } \\
\text { time }\end{array}$ \\
\hline
\end{tabular}

\section{DEEP LEARNING METHODS OF PNEUMONIA DETECTION WITH CHEST X-RAY IMAGES}

Deep learning approaches have gained popularity as a result of significantly increased frame categorization effectiveness. The organization's activation functions, together with classifiers, aid in retrieving temporal and spatial characteristics from frames. A weight-sharing mechanism is used in the levels, which significantly reduces computing time.

\subsection{CNN (Convolutional NN)}

Convolutional neural networks are essentially back propagation computational models with additional restrictions such as terminals in almost the same filtration system are only linked to small sections of something like the frame to maintain geographic distribution, and individual strengths are distributed to significantly reduce the number of hyper parameters. A CNN is made up of multiple layers: 


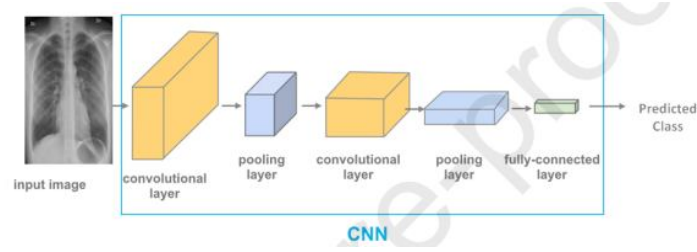

Figure 2: Architecture of CNN [4]

The first layer is the convolutional layer that provides categorization functions to the structure [4]. The other layer feature map is to subsequent development, the next layer is subsampling (max-pooling) layer to downward sampling the frame, minimize dimensions, and reducing computation complexity. The architecture of CNN is shown in above figure 2. The several layers of $\mathrm{CNN}$ are discussed below:

Convolutional layer: In the convolutional layer, the matrix is formed by images. The feature map has resulted in this layer after the combination of feature kernel and image matrix. The main objective of this layer is to reduce the feature dimensions of the image. In some cases, the reduction of feature dimension resulted in the loss of essential information. To reduce this loss various feature detectors are used inside this layer.

Activation function: The activation function is used for the better performance of the model. There are various activation functions such as softmax, ReLU, sigmoid, etc. The ReLu is the activation function that is used mostly in all models. It provides the feature map with a linear function. Softmax is based on the theory of probability distribution.

Pooling layer: The main objective of this layer is dimension reduction. The pooling layer is also used to reduce the computational complexity by dimension reduction. For computational complexity, sampling partition is used and essential salient features are also detected in this layer.

Fully connected layers: In this layer, the image is passed through the fully connected layer. The cost function is defined in this layer after the evaluation of weight calculation. The performance of the model is based on the cost function.

\subsection{VGG16 and VGG19 Network}

Simonyan and A. Zisserman were created the VGG16, which is a CNN model. It was among the most well-known designs entered in the 2014 ILSVRC challenge. On the Imagenet database, the VGG model obtains a top-five prediction performance of 92.7 per cent. The region connects sixteen levels in aggregate. Numerous 3X3 operator filtrations were implemented next to each other in VGG16; eliminating massive operator filtering that was utilized in traditional approaches. The artificial network's capacity is boosted by using many levels of neurons. The artificial network's thickness is boosted by using many levels of neurons. This allows the neural net to recognize and grasp more complicated issues and characteristics.

Vgg16 has $3 \times 3$ dimensional activation functions, $2 \times 2$ dimensional ordinary layers, and a fully linked layer upon layer. The neural network's starting dimension is 64 . After every max pooling, the neuronal network's size increases. Each of the other two completely activation functions contains 256 stations, whereas the network structure only has two. The ReLU objective function is used in the initial multiple convolutional layers, while the nonlinear activation neural network is used in the output layer. After each 256 connection hidden layers, dropping is implemented. The channel's activation function used in VGG16 is 0.0001 . Employing categorization inter as the covariance matrix, the optimizer Adam was employed. VGG16's descriptive complexity is advantageous for prediction performance [10]. Vgg19 is a nineteen-layer CNN (convolutional neural network) that is mostly used during object recognition. It is a variation of VGG16. It has an identical overall concept to VGG16. Only one distinction in VGG19 is that it employs two large amounts with 256 and two channels, as well as connection weights of 0.00001 . The VGG-16 architecture is represented in figure 3.

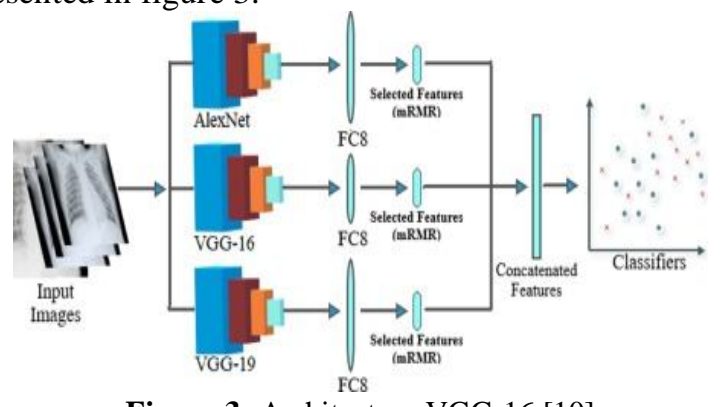

Figure 3: Architecture VGG-16 [10]

\subsection{ResNet50 Network}

ResNet50 is a type of image categorization algorithm. In 2015, Microsoft's ResNet secured the ILSVRC categorization challenge with a top 5 failure rate of 3.57 per cent upon on ImageNet dataset. The platform's fully connected layers have $3 X 3$ filtration and the pooling layer with a stride of 2 down sample immediately. The platform's output layer is a reasonably obtainable structure containing 256 and two channels, using ReLU and softmax activation algorithms. The platform's activation function is 0.000001 . Employing categorizing bridge as the optimization problem, the Adam optimizer was employed [11].

ResNet employs shortcut connections to address the issues of degraded precision and disappearing elevation that plague DNN (deep neural networks). These links enable the customers to pass across levels that it deems unimportant during development [17]. There are multiple types of ResNet34, ResNet50, ResNetV2, ResNetxt. There are fifty layers in ResNet50 that are used for the detection and classification of $\mathrm{x}$-rays images. The ResNet50 is represented in figure 4 . 


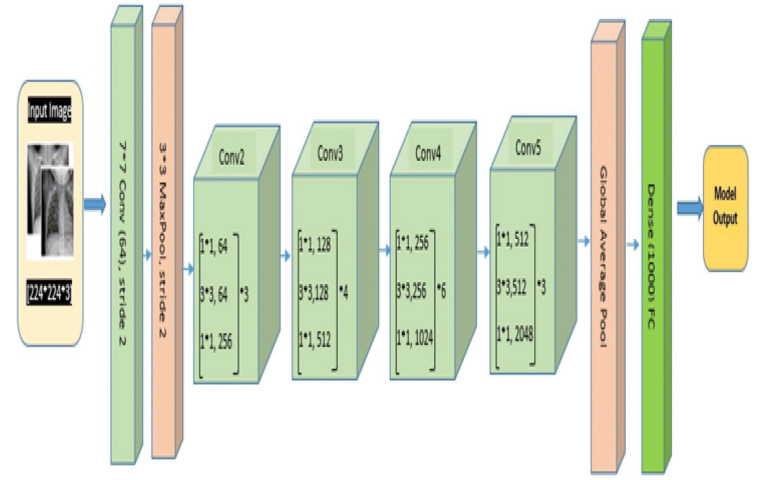

Figure 4: Architecture of ResNet50 [11]

\subsection{Inception-v4}

Shallow Convolutional Neural Network (CNN) architectures are essential for one of the most advanced breakthroughs in image classification. Inception has become one of the architectures that display satisfactory accuracy at a reasonable cost of processing. The Inception deep network design is also known as Inception-v1.

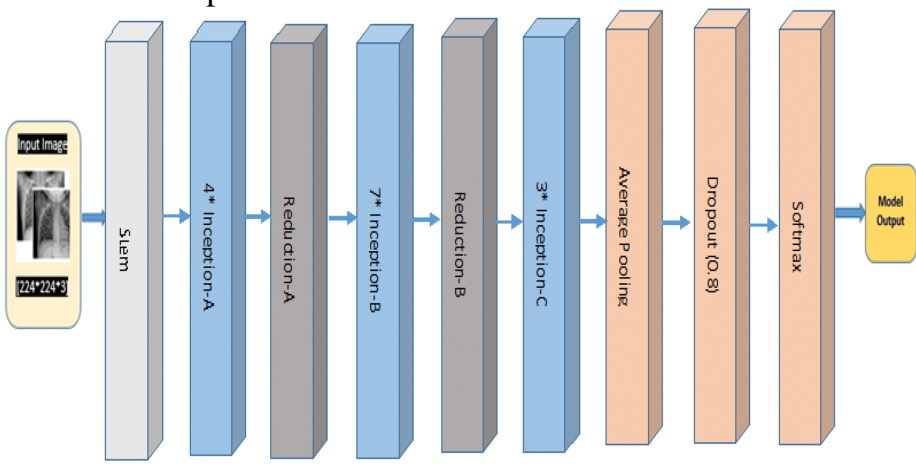

Figure 5: Architecture of Inception-V4 [12]

The Inception-v1 framework with the theory of residual blocks is resulting in Inception-v2. Then, in subsequent versions, the decomposition concept is incorporated, as well as the framework is dubbed Inception ResNet V1, Inception-v1, Inception ResNet V2, Inception-v3, and Inception-v4 [12]. The framework of InCeptionV4 is shown in above fig. 5. The comparative analysis with different deep learning methods is shown in Table 3.

\subsection{Transfer Learning with CNN}

Convolutional neural networks are widely used in recent image processing high-dimensional data (CNNs). Most layers explicitly assume that every data they receive is a bitmap. Initially, the convolution layer is composed in a fine aggregate visual and finds ways to recognize decreased characteristics like boundaries. Several layers are effective in controlling an object's temporal and spatial correlations. Filtrations are used to do this. These layers, contrast traditional feed-forward folds, feature a substantially smaller set of variables, and use a weight-sharing mechanism to save calculation time [3]. The different deep learning methods layers are mentioned in Table 4.
The employment of similar pre-trained algorithms is essential rather than engaging through to the complicated process of classification algorithms from the start when embarking on a comparable image recognition challenge. Transferring learning refers to the process of emigrated through one present and training system to a web application through recycling the different network parameters [13]. The deep transfer learning architecture is presented in figure 6 .

The Hyper-parameters used while fine-tuning the deep learning models are presented in Table 5.

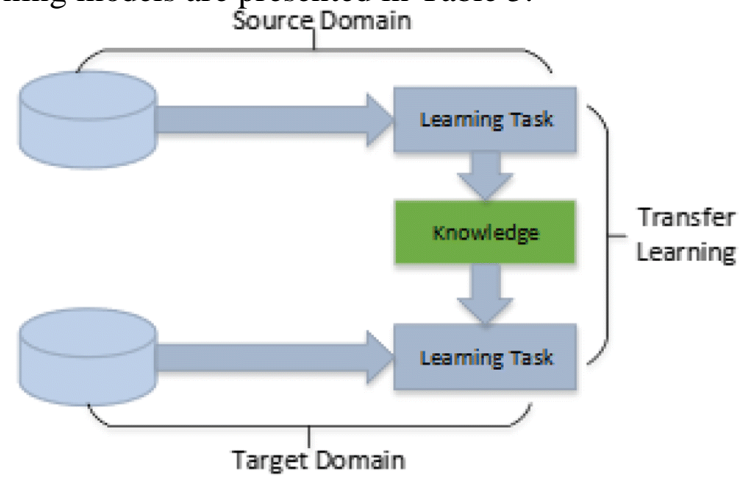

Figure 6: Architecture of transfer learning [13]

Below the table 3, 4, and 5 described the comparative number of parameters, layers and Hyper-parameters used while fine-tuning the deep learning models.

Table 3: Comparative number of parameters with different deep learning methods

\begin{tabular}{|l|l|}
\hline Methods & Number of Parameters \\
\hline CNN & 62 millions \\
\hline RestNet 50 & 23 millions \\
\hline Vgg19 & 144 millions \\
\hline Vgg16 & 138 millions \\
\hline Transfer Learning & - \\
\hline Inception-4 & 5 millions \\
\hline
\end{tabular}

Table 4: Different deep learning methods: Layers

\begin{tabular}{|l|l|}
\hline Methods & Layers \\
\hline CNN & 6 \\
\hline RestNet 50 & 50 \\
\hline Vgg19 & 19 \\
\hline Vgg16 & 16 \\
\hline Transfer Learning & 9 \\
\hline Inception-4 & 22 \\
\hline
\end{tabular}

Table 5: Hyper-parameters used while fine-tuning the deep learning models

\begin{tabular}{|c|c|c|c|c|c|c|}
\hline $\begin{array}{l}\text { Arc } \\
\text { hite } \\
\text { ctur } \\
\text { e }\end{array}$ & $\begin{array}{l}\text { Ima } \\
\text { ge } \\
\text { Size }\end{array}$ & $\begin{array}{l}\text { Optimiz } \\
\text { er }\end{array}$ & $\begin{array}{l}\text { Epo } \\
\text { chs }\end{array}$ & $\begin{array}{l}\text { Lear } \\
\text { ning } \\
\text { Rate }\end{array}$ & $\begin{array}{l}\text { Momentu } \\
\text { m }\end{array}$ & $\begin{array}{l}\text { Wei } \\
\text { ght } \\
\text { Dec } \\
\text { ay }\end{array}$ \\
\hline $\begin{array}{l}\text { Res } \\
\text { Net5 } \\
0\end{array}$ & $\begin{array}{l}224 * \\
224\end{array}$ & $\begin{array}{l}\text { Stochast } \\
\text { ic } \\
\text { Gradient } \\
\text { Descent }\end{array}$ & 50 & $\begin{array}{l}0.000 \\
1\end{array}$ & 0.9 & $\begin{array}{l}0.00 \\
01\end{array}$ \\
\hline
\end{tabular}




\begin{tabular}{|l|l|l|l|l|l|l|}
\hline $\begin{array}{l}\text { Ince } \\
\text { ptio } \\
\text { nV3 }\end{array}$ & $\begin{array}{l}229 * \\
229\end{array}$ & $\begin{array}{l}\text { Stochast } \\
\text { ic } \\
\text { Gradient } \\
\text { Descent }\end{array}$ & 25 & 0.001 & 0.9 & $\begin{array}{l}0.00 \\
1\end{array}$ \\
\hline $\begin{array}{l}\text { Vgg } \\
16\end{array}$ & $\begin{array}{l}224 * \\
224\end{array}$ & Adam & 16 & 0.001 & 0.9 & $\begin{array}{l}0.00 \\
1\end{array}$ \\
\hline $\begin{array}{l}\text { Vgg } \\
19\end{array}$ & $\begin{array}{l}224 * \\
224\end{array}$ & Adam & 19 & 0.001 & 0.9 & $\begin{array}{l}0.00 \\
1\end{array}$ \\
\hline
\end{tabular}

The graphical representations of different existing techniques are shown in figure 7 and figure 8.

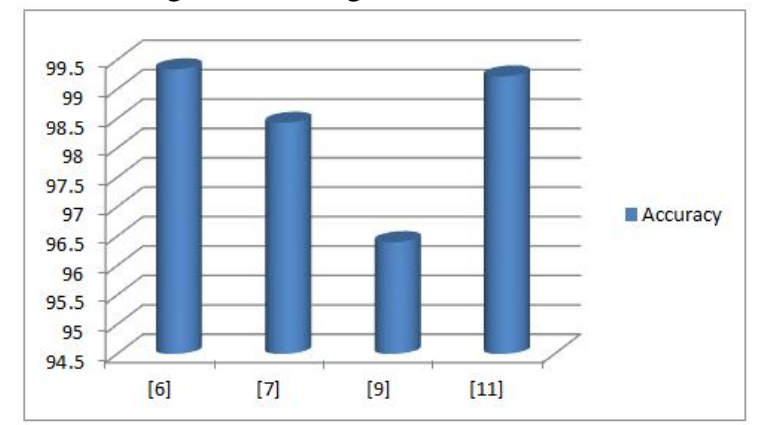

Figure 7: Comparison between existing techniques based on accuracy

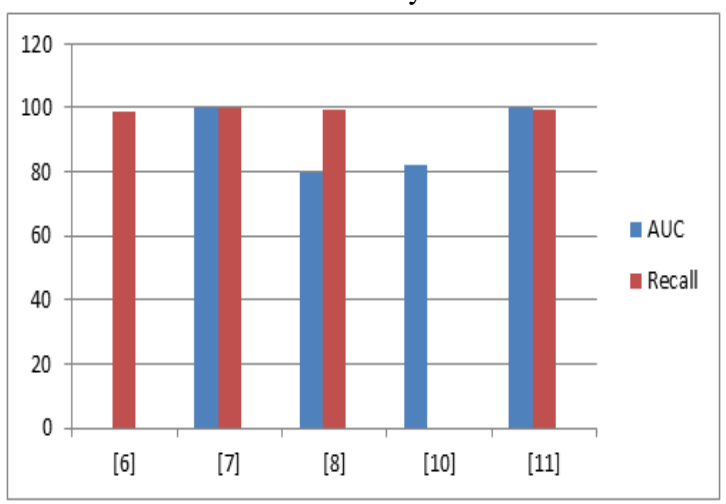

Figure: 8 Graphical representations of various existing techniques based on different parameters

\section{CONCLUSION AND FUTURE WORK}

Pneumonia seems to be a viral infection that can be treated in advanced nations which is often devastating in underdeveloped countries. In this paper, deep learning based pneumonia detection existing techniques are surveyed. The limitations of existing methods are also discussed in Table1. There are different types of causes and symptoms are disused in this paper. Various deep learning based pneumonia detection frameworks are discussed. The main method of deep learning is convolutional neural network is also explained in this paper. The VGG16 and VGG19 advance deep neural networks are also discussed in paper. Each model has its own way of computation. ResNet used fifty layers whereas VGG-16 used 16 layers for computation. Shallow CNN based Inception-V4 model is also depicted in this paper. The further work can implement a novel hybridization approach to detect the disease and improve the performance.

\section{REFERENCES}

1. Stephen, O., Sain, M., Maduh, U. J., \& Jeong, D. U., An efficient deep learning approach to pneumonia classification in healthcare. Journal of healthcare engineering, 2019.

2. Varshni, D., Thakral, K., Agarwal, L., Nijhawan, R., \& Mittal, A., Pneumonia detection using CNN based feature extraction. In 2019 IEEE International Conference on Electrical, Computer and Communication Technologies (ICECCT), pp. 1-7, February 2019.

3. Madhavi K, Madhavi G, Devi Rupa B , Kora Padmavathi., Detection of Pneumonia Using Deep Transfer Learning, International Journal of Advanced Trends in Computer Science and Engineering, Vol. 9, No.5, 2020

4. Rahman, T., Chowdhury, M. E., Khandakar, A., Islam, K. R., Islam, K. F., Mahbub, Z. B., ... \& Kashem, S., Transfer learning with deep convolutional neural network (CNN) for pneumonia detection using chest X-ray. Applied Sciences, Vol. 10, pp. 3233, 2020

5. Sekeroglu, B., \& Ozsahin, I., <? covid19?> Detection of COVID-19 from Chest X-Ray Images Using Convolutional Neural Networks. SLAS TECHNOLOGY: Translating Life Sciences Innovation, Vol. 25, pp. 553-565, 2020

6. Hashmi, M. F., Katiyar, S., Keskar, A. G., Bokde, N. D., \& Geem, Z. W., Efficient pneumonia detection in chest xray images using deep transfer learning. Diagnostics, Vol.10, pp. 417, 2020

7. Chouhan, V., Singh, S. K., Khamparia, A., Gupta, D., Tiwari, P., Moreira, C., ... \& De Albuquerque, V. H. C., A novel transfer learning based approach for pneumonia detection in chest X-ray images. Applied Sciences, Vol. 10 , pp. 559, 2020

8. Acharya, A. K., \& Satapathy, R., A deep learning based approach towards the automatic diagnosis of pneumonia from chest radio-graphs. Biomedical and Pharmacology Journal, Vol. 13, pp. 449-455, 2020

9. Elshennawy, N. M., \& Ibrahim, D. M., Deep-Pneumonia Framework Using Deep Learning Models Based on Chest X-Ray Images. Diagnostics, Vol.10, pp. 649, 2020

10. Jain, R., Nagrath, P., Kataria, G., Kaushik, V. S., \& Hemanth, D. J., Pneumonia detection in chest X-ray images using convolutional neural networks and transfer learning. Measurement, Vol. 165, pp. 108046, 2020

11. Toğaçar, M., Ergen, B., Cömert, Z., \& Özyurt, F., A deep feature learning model for pneumonia detection applying a combination of $\mathrm{mRMR}$ feature selection and machine learning models. Irbm, Vol. 41, pp. 212-222, 2020

12. Phankokkruad, M., COVID-19 Pneumonia Detection in Chest X-ray Images Using Transfer Learning of Convolutional Neural Networks. In Proceedings of the 3rd International Conference on Data Science and Information Technology pp. 147-152, 2020 
13. Luján-García, J. E., Yáñez-Márquez, C., Villuendas-Rey, Y., \& Camacho-Nieto, O, A transfer learning method for pneumonia classification and visualization. Applied Sciences, Vol. 10, pp. 2908, 2020

14. Pneumonia Symptoms and Diagnosis. (2021). Retrieved 27 May 2021, from https://www.lung.org/lung-health-diseases/lung-disease-1 ookup/pneumonia/symptoms-and-diagnosis

15. Pneumonia - Symptoms and causes. (2021). Retrieved 27 May 2021, from https://www.mayoclinic.org/diseases-conditions/pneumo nia/symptoms-causes/syc-20354204

16. Rajasenbagam, T., Jeyanthi, S., \& Pandian, J. A., Detection of pneumonia infection in lungs from chest $\mathrm{X}$-ray images using deep convolutional neural network and content-based image retrieval techniques. Journal of Ambient Intelligence and Humanized Computing, pp.1-8,2021

17. Angeline, R., \& Vani, R., ResNet: A convolutional Neural Network for detecting and diagnosing of coronavirus pneumonia. In IOP Conference Series: Materials Science and Engineering Vol. 1084, No. 1, p. 012011, March 2021. 